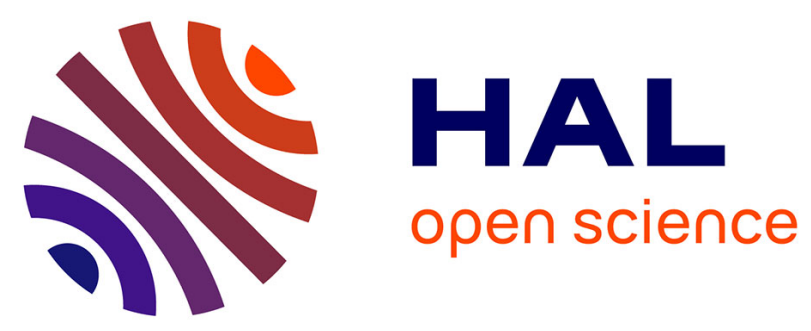

\title{
Elaboration d'un indice de complexité des éditeurs d'équations: L'exemple de la comparaison de HandiMathKey et Libreoffice
}

Jean-Marc Meunier, Rozenn Baudet, Frédéric Vella, Nadine Vigouroux

\section{- To cite this version:}

Jean-Marc Meunier, Rozenn Baudet, Frédéric Vella, Nadine Vigouroux. Elaboration d'un indice de complexité des éditeurs d'équations: L'exemple de la comparaison de HandiMathKey et Libreoffice. 32ème Conférence francophone sur l'Interaction Humain-Machine (IHM 2021), Apr 2021, Virtual Event, France. pp.1-6, 10.1145/3451148.3458640 . hal-03562163

\section{HAL Id: hal-03562163 https://hal.science/hal-03562163}

Submitted on 8 Feb 2022

HAL is a multi-disciplinary open access archive for the deposit and dissemination of scientific research documents, whether they are published or not. The documents may come from teaching and research institutions in France or abroad, or from public or private research centers.
L'archive ouverte pluridisciplinaire HAL, est destinée au dépôt et à la diffusion de documents scientifiques de niveau recherche, publiés ou non, émanant des établissements d'enseignement et de recherche français ou étrangers, des laboratoires publics ou privés. 


\title{
Élaboration d'un indice de complexité des éditeurs d'équations : L'exemple de la comparaison de HandiMathKey et Libreoffice
}

\author{
Elaboration of an equation editor complexity index: Example of the comparison between \\ HandiMathKey and Libre Office \\ Jean-Marc Meunier* \\ Laboratoire Paragraphe, Université Paris 8 \\ Frédéric Vella \\ IRIT, UMR CNRS 5505, Université Paul Sabatier \\ Rozenn Baudet \\ Laboratoire Paragraphe, Université Paris 8 \\ Nadine Vigouroux \\ IRIT, UMR CNRS 5505, Université Paul Sabatier
}

\begin{abstract}
Pupils with a grapho-motor disorder have difficulties with handwriting]. To compensate for these problems, the use of a computer is recommended but is not suitable for inputting mathematical formulae. A new software called "HandiMathKey" (HMK) has been developed to address the difficulties encountered. In order to determine whether this tool is efficient, we compared HMK and Libre Office (LO) with pupils from the sixth grade in secondary school. In order to make this comparison, standard methods (time, success...) have limitations and do not allow for a specific comparison of tasks and editors between them, nor of the procedures performed by the participants. We have therefore carried out an in-depth analysis of the task and defined variables that allow a cognitive evaluation of the activity. Based on this analysis, we were able to design a complexity index allowing the comparison of the editors but also of the participants' actual tasks and procedures. The design of this index is innovative and provides new possibilities of measurement for the study of artefact instrumentalization.
\end{abstract}

\section{CCS CONCEPTS}

- Human-centered computing $\rightarrow$ Human computer interaction (HCI); HCI design and evaluation methods; User studies.

\section{KEYWORDS}

Cognitive evaluation, activity, complexity index, software comparisonl

\section{RÉSUMÉ}

Les élèves présentant un trouble grapho-moteur rencontrent des difficultés lors de l'écritures manuscrite. Pour compenser ces troubles, l'usage d'un ordinateur est recommandé mais s'avère inadapté pour la saisie de formules mathématiques. Un nouveau logiciel nommé «HandiMathKey »(HMK) a été conçu dans l'objectif de répondre aux difficultés de saisie rencontrées. Afin de déterminer si cet outil est efficient, nous avons comparé HMK et Libre Office (LO) auprès d'élèves scolarisés en sixième. Pour faire cette comparaison, les méthodes standard (temps, réussite. . .) présentent des limites et ne permettent pas de comparer spécifiquement les tâches et les éditeurs entre eux, ni les procédures effectuées par les participants. Nous avons donc réalisé une analyse approfondie de la tâche et défini des variables qui permettent une évaluation cognitive de l'activité. A partir de cette analyse, nous avons pu concevoir un indice de complexité permettant la comparaison des éditeurs mais aussi des tâches et procédures effectives des participants. La conception de cet indice est innovante et ouvre des possibilités nouvelles de mesure pour l'étude sur l'instrumentalisation d'artefact.

\section{MOTS-CLÉS}

Evaluation cognitive, activité, indice de complexité, comparaison de logicie

\section{ACM Reference Format:}

Jean-Marc Meunier*, Rozenn Baudet, Frédéric Vella, and Nadine Vigouroux. 2021. Élaboration d'un indice de complexité des éditeurs d'équations : L'exemple de la comparaison de HandiMathKey et Libreoffice: Elaboration of an equation editor complexity index: Example of the comparison between HandiMathKey and Libre Office. In 32e Conférence Francophone sur l'Interaction Homme-Machine (IHM '21 Adjunct), April 13-16, 2021, Virtual Event, France. ACM, New York, NY, USA, 6 pages. https: //doi.org/10.1145/3451148.3458640

\section{INTRODUCTION}

Les élèves présentant des troubles grapho-moteurs (dysgraphique ou trouble de l'attention avec ou sans hyperactivité ou TDAH) rencontrent des difficultés lors de l'écriture manuscrite [1,2]. Les élèves porteurs d'une dysgraphie présentent une atteinte des processus sensorimoteurs. A l'inverse, les élèves porteurs d'un TDAH voient la réalisation des gestes moteurs impactés en raison d'une grande charge mentale [3]. Pour compenser ces troubles, l'usage d'un ordinateur est recommandé mais s'avère inadapté pour la saisie de formules mathématiques. Les raisons avancées sont (i) la complexité de cette saisie dans les éditeurs de traitement de text [3] (ii) le coût cognitif, du fait de la superposition des espaces de saisie et le passage de l'un à l'autre $[4,5]$. Plus précisément, ont été rapportés des difficultés de repérages sur l'éditeur, le manque d'intuitivité, la forte sollicitation d'attention visuo-spatiale et motrice, les nombreux déplacements souris, le temps plus long pour copier les formules. Ces difficultés constituent un «sur-handicap »qui contraint ces élèves 
à revenir à une écriture manuscrite durant les cours de mathématiques alors que celle-ci n'est pas performante [6, 7].

C'est pour résoudre ces difficultés que le clavier HandiMathKey (HMK) a été co-conçu [8]. Il se présente comme un clavier virtuel réunissant les symboles mathématiques utilisés dans l'enseignement secondaire. HMK s'avère adapté aux besoins spécifiques des utilisateurs [4], mais il n'existe aucune comparaison avec d'autres éditeurs d'équations. C'est ce que nous avons entrepris en comparant l'efficience de HMK et de l'éditeur d'équations de LibreOffice (LO) en fonction de la charge cognitive dans une tâche de copie de formules par des élèves de collège.

\section{MESURER L'EFFICIENCE EN FONCTION DE LA CHARGE COGNITIVE}

Les modes de réalisation d'une formule sont très variables pour un même éditeur et d'un éditeur à un autre. Les mesures habituelles en termes de temps de réalisation ou de réussite s'avèrent donc insuffisantes pour rendre compte finement des effets du dispositif sur l'activité. Dans le champ de la saisie de textes, [9] a proposé le KSPC (Keystrokes Per Character, le nombre de frappes par caractère) comme métrique pour évaluer les claviers virtuels de saisie de textes. Une autre mesure consiste à considérer la vitesse de saisie à condition de considérer la longueur des mots ou la complexité de ce qui doit être saisi [10]. La mesure de l'utilisabilité et de l'ergonomie $\mathrm{du}$ logiciel de saisie à l'aide de questionnaires de perception [11] s'avère également peu praticable avec des enfants, surtout déficient cognitif. Une telle mesure porte en outre sur la perception du logiciel et non la performance du sujet que nous cherchons à appréhender dans ce travail. Dans notre cas, les éditeurs d'équations présentent plusieurs caractéristiques communes qui sont sources d'un accroissement de la charge cognitive. (i) La saisie de formule se fait dans une fenêtre distincte de celle de l'éditeur de texte ce qui impose des changements fréquents du focus ; (ii) elle nécessite alternativement le clavier physique, la souris ou le clavier virtuel, ce qui impose également une distribution des ressources attentionnelles ; (iii) elle nécessite une décomposition et une planification de l'activité qui peut conduire, en fonction de l'expertise à une grande variabilité du nombre d'actions nécessaires. Pour analyser les tâches, nous avons considéré l'usage des logiciels sous l'angle de la résolution de problèmes et analysé les tâches en tant que tel en essayant (1) de caractériser l'espace de la tâche et (2) l'évolution du sujet dans cette espace [12]. La notion d'espace de la tâche renvoie à l'ensemble des cheminements entre la situation initiale et le but. De ce point de vue, une procédure sera d'autant plus complexe qu'elle nécessitera un nombre important d'actions. La complexité dont nous parlons ici est une complexité procédurale par opposition à une complexité sémantique ou conceptuelle [13] qui demanderons des mesures complémentaires. La définition d'une métrique de la complexité procédurale n'en est pas moins importante parce qu'elle est, s'agissant de dispositifs informatiques, automatisable et surtout généralisable à d'autres dispositifs de communication. Une telle mesure permet en outre d'appréhender, même si c'est de manière indirecte, les processus cognitifs à l'œuvre dans la réalisation de la tâche $[14,15]$ et comme nous allons le développer l'apprennabilité du dispositif.

\section{DEFINITION D'UN INDICATEUR DE COMPLEXITE}

Pour faire la comparaison entre les éditeurs de formule HMK et LO, nous avons donc réalisé une analyse poussée de la tâche ou plutôt des tâches. Nous avons élaboré une série de tâches qui permettaient d'explorer les différents menus et sous menus des éditeurs. Ensuite, nous avons répertorié l'ensemble des actions nécessaires pour mener à bien chacune des tâches. Cette analyse nous a permis d'identifier les procédures optimales, c'est-à-dire les plus courtes et les procédures les moins optimales, c'est-à-dire nécessitant le plus d'actions sans retour en arrière. Elles ont été listées dans un tableau à partir duquel ont pu être extraites et définies des variables qui permettent la comparaison intertâches, inter et intrasujet en nous inspirant des travaux de Lee et al. [16] et Gabadinho et al. [17]. L'idée de base est que la complexité d'une tâche dépend principalement du nombre d'opérations nécessaires pour la réaliser. Certaines opérations nécessitent plus que d'autres un changement $\mathrm{du}$ focus attentionnel, nous proposons donc de les distinguer. Pour chacun des éditeurs, nous avons identifié les actions à effectuer pour réaliser chaque tâche de copie d'une formule. Nous avons ensuite comptabilisé le nombre d'actions nécessaires $\left(\mathrm{N}_{\mathrm{A}}\right)$ (mouvements de souris entre deux cibles, clics souris, appuis clavier), le nombre de switch clavier physique /souris $\left(\mathrm{N}_{\mathrm{S}}\right)$ et le nombre de changements de niveau et/ou de fenêtre $\left(\mathrm{N}_{\mathrm{F}}\right)$. Chacune de ces variables est ensuite pondérée par le nombre de caractère à saisir $\left(\mathrm{N}_{\mathrm{C}}\right)$ afin de calculer un indice de complexité de la planification des actions ( $\left.\mathrm{IC}_{\mathrm{A}}\right)$, de la gestion des fenêtres $\left(\mathrm{IC}_{\mathrm{F}}\right)$ et des switches $\left(\mathrm{IC}_{\mathrm{S}}\right)$. La somme de ces trois rapports constitue un indice de complexité globale $\left(\mathrm{IC}_{\mathrm{G}}\right)$ de la formule à saisir. Nous avons donc :

$$
I C_{G}=I C_{A}+I C_{F}+I C_{S} \text { autrement dit IC } C_{G}=\frac{N_{A}}{N_{C}}+\frac{N_{F}}{N_{C}}+\frac{N_{S}}{N_{C}}
$$

\section{COMPARABILITE DES TACHES}

La complexité d'une procédure de saisie sur un éditeur d'équations est variable pour une même formule et un même éditeur. Nous pouvons donc définir la procédure optimale $\left(\mathrm{IC}_{\mathrm{G}}\right.$ minimum), et la procédure la plus longue ( $\mathrm{IC}_{\mathrm{G}}$ maximum). L'ensemble des procédures possibles pour une même formule est ainsi ordonnable sur $\mathrm{IC}_{\mathrm{G}}$ et constitue l'espace de la tâche dans laquelle le sujet peut évoluer. Nous pouvons quantifier l'importance de l'espace de la tâche calculant la différence entre le maximum et le minimum. En le rapportant au maximum, nous obtenons un écart relatif $\mathrm{ER}_{\mathrm{T}}$ qui autorise la comparaison entre tâches du point de vue de la variabilité des procédures. Cet écart varie entre 0 (lorsque maximum est égal au minimum) et 1 (cas purement théorique où la complexité minimale est de 0 ).

$$
E R_{T}=\frac{I C_{\max }-I C_{\min }}{I C_{\max }}
$$

Dans le cas d'HMK, une seule procédure est possible, $\mathrm{ER}_{\mathrm{T}}$ (1) est donc égale à 0 pour toutes les tâches. $\mathrm{ER}_{\mathrm{T}}$ pour $\mathrm{LO}$ s'interprète donc comme le surcroît de complexité par rapport à HMK. Dans la tâche $1, \mathrm{ER}_{\mathrm{T} 1}=0,46$, ce qui veut dire que la tâche 1 dans $\mathrm{LO}$ est 0,46 fois plus complexe que dans HMK. Nous pouvons observer dans le tableau 1 que l'ordre des valeurs pour $\mathrm{ER}_{\mathrm{T}}$ diffère de celui de $\mathrm{IC}_{\mathrm{G}}$, nous reviendrons sur cet élément dans la partie résultat. 
Élaboration d'un indice de complexité des éditeurs d'équations : L'exemple de la comparaison de HandiMathKey et Libreoffice

Table 1: Indices de complexité globale par éditeur et par tâche et écarts relatifs (ERT) par tâche

\begin{tabular}{|c|c|c|c|c|}
\hline Tâches & $\mathrm{IC}_{\mathrm{G}}$ & & & $\mathrm{ER}_{\mathrm{T}}$ \\
\hline & HMK & $\mathrm{LO}_{\min }{ }^{1}$ & $\mathrm{LO}_{\max }{ }^{2}$ & LO \\
\hline $\mathrm{T} 1: V=\pi(2 R)^{3}=\pi 2^{3} R^{3}=\pi 8 R^{3}$ & 3 & 5,4 & 10 & 0,466 \\
\hline $\mathrm{T} 2: \mathrm{A}_{2}=\frac{3 \times 7,5}{2}=11,25 \mathrm{~m}^{2}$ & 2,88 & 3,3 & 5,7 & 0,412 \\
\hline $\left.\mathrm{T} 3: \mathrm{D}=\overline{\widehat{A W H}}=\sin ^{-1}\left(\frac{8,6}{10,6}\right) \approx 54,2^{\circ}\right\}$ & 2,58 & 4,4 & 5,1 & 0,131 \\
\hline $\mathrm{T} 4: \mathrm{A}=\frac{-3}{5} \div\left(\frac{13}{8}-\frac{-8}{5}\right)$ & 2,68 & 4,7 & 5,1 & 0,079 \\
\hline $\mathrm{T} 5: \mathrm{B}=\sqrt{D A^{2}-D K^{2}}=\sqrt{60^{2}-11^{2}}=\sqrt{3479} \approx 59,0 \mathrm{~cm}$ & 3,1 & 4,1 & 6,1 & 0,333 \\
\hline $\mathrm{T} 6: \mathrm{E}=\frac{1}{4}+1^{8}=1,25$ & 3,53 & 3,9 & 7,1 & 0,446 \\
\hline $\mathrm{T} 7: \mathrm{C}=(2 \sqrt{6}-5 \sqrt{10})^{2}=(2 \sqrt{6})^{2}-2 \times 2 \sqrt{6} \times 5 \sqrt{10}+(5 \sqrt{10})^{2}$ & 2,13 & 4,8 & 8,1 & 0,407 \\
\hline
\end{tabular}

\section{PROTOCOLE EXPERIMENTAL}

\subsection{Population}

Quatorze élèves (8 garçons et 6 filles) scolarisés en sixième, répartis en trois groupes (Groupe 1 : Tout-venant $(\mathrm{N}=2)$; Groupe 2 : Dysgraphique et faible scripteur $(\mathrm{N}=6)$ et Groupe 3 : Trouble Déficit de l'Attention/Hyperactivité (TDAH) $(\mathrm{N}=6)$ ont pu être recrutés. Le consentement libre et éclairé a été demandé aux parents. La notice explicative de la recherche a été transmise avant la passation. La famille a été informée de son droit de se retirer de l'étude à tout moment ainsi que de la garantie de l'anonymat.

\subsection{Passation}

Dans chacun des trois groupes la moitié des élèves ont réalisé la saisie des formules mathématiques avec HMK, l'autre moitié avec LO. Lors de l'étude exploratoire [10], les sept tâches (notées T1 à T7) ont été présentées dans un ordre fondé sur le critère de complexité que nous avons abandonné depuis mais que nous indiquons ici pour permettre de comprendre ce qui a été fait. Cet indice, que nous nommons ici IG (2), était calculé à partir des moyennes de la valeur maximale et minimale, pour les deux logiciels, du nombre d'actions $\left(\mathrm{N}_{\mathrm{A}}\right)$, de changement de fenêtres $\left(\mathrm{N}_{\mathrm{F}}\right)$ et de switchs $\left(\mathrm{N}_{\mathrm{c}}\right)$, pondérées par le nombre de caractères à saisir dans l'éditeur. La formule est la suivante :

$$
\begin{aligned}
I G= & \frac{\text { Moyenne }\left(N_{\text {Amax }+ \text { min }}\right)}{N_{C}} \\
& +\frac{\text { Moyenne }\left(N_{\text {Fmax }}+\min \right)}{N_{C}} \\
& +\frac{\text { Moyenne }\left(N_{C \max +\min }\right)}{N_{C}}
\end{aligned}
$$

Cet indice général de complexité a permis de présenter les tâches par ordre de complexité croissante (allant de 3,18 à 5). Le présent article permet de voir l'évolution de notre réflexion sur cet indice de complexité.

Les passations se sont déroulées de manière individuelle au domicile de chaque participant avec un ordinateur spécifique pour l'étude équipé de LO, d'HMK et d'un enregistreur vidéo. Il a été demandé à chaque participant de recopier sept formules mathématiques avec le logiciel HMK ou Libre Office que les participants découvraient alors. Aucune formation pour les deux outils n'a été proposé.

\section{RESULTAT}

\subsection{Comparabilité des sujets}

Il est possible de caractériser la performance des sujets par un écart relatif $\left(E R_{S}\right)$ (3) entre la complexité de la procédure observée $\mathrm{IC}_{\mathrm{obs}}$ et la procédure optimale $\mathrm{IC}_{\min }$ pour chacun des types de complexité avec un simple rapport :

$$
E R_{s}=\frac{I C_{o b s}-I C_{\text {min }}}{I C_{o b s}}
$$

Une telle transformation constitue une mesure de l'écart entre la performance du sujet et la procédure optimale. Cela permet de ramener sur une même échelle l'ensemble des indices de complexité. On peut ainsi les comparer. Il devient alors possible d'étudier les différences de performance sur des tâches de complexités différentes et donc de comparer les éditeurs sur une variété de tâches mais également de faire des comparaisons intergroupes sur tout ou partie du protocole. Lors de l'analyse des résultats, il a été observé la présence d'écarts relatifs négatifs et une variation faussement importante en raison des abandons en cours de tâche ou omission de caractères. Ces écarts relatifs négatifs après comparaison avec les fichiers de traces écrites des participantes correspondent à des tâches non terminées ou différentes de la tâche prescrite. Ainsi, il apparaît que de nombreux participants n'ont pas correctement recopié la tâche, induisant une diminution des actions, switchs et changements de fenêtre plus ou moins importante. Dès lors, nous avons exclu les données concernées de l'analyse. Le nombre de participants dont les résultats ont pu être pris en compte dans le calcul des $\mathrm{ER}_{\mathrm{T}}$ et de la variation de la progression à la tâche figure dans le tableau 2. Ainsi le calcul de l'ER $\mathrm{T}_{\mathrm{T}}$ constitue-t-il un outil permettant de repérer rapidement les sujets qui ont potentiellement abandonné en cours de tâche?

L'analyse des données résumée dans le tableau 2 nous permet de constater une différence systématique entre les écarts relatifs à la procédure optimale en faveur de HMK comparativement à Libre Office. Cette différence allant de 0,7 points pour la tâche 6 jusqu'à 3,2 points à la tâche 7 .

\subsection{Comparabilité des progressions}

Pour les comparaisons intra sujets, on peut également utiliser $\mathrm{ER}_{\mathrm{S}}$ (4) dans des procédures statistiques à mesures répétées, mais il est plus intéressant de calculer à partir des écarts relatifs un taux de variation et ainsi quantifier la variation de l'écart à la procédure 
Table 2: Nombre de sujets, moyennes et écarts-types des écarts relatifs ERS des sujets par éditeur

\begin{tabular}{lllllll}
\hline ER des tâches & \multicolumn{2}{l}{ Nombre de sujets } & \multicolumn{2}{l}{ HMK } & \multicolumn{2}{l}{ LO } \\
\cline { 2 - 7 } & HMK & LO & Moyenne & Ecart-Type & Moyenne & Eart-Type \\
\hline T1 & 5 & 6 & 7,97 & 2,36 & 11 & 3,13 \\
T2 & 7 & 7 & 6,08 & 2,31 & 9,2 & 3,88 \\
T3 & 7 & 5 & 4,77 & 1,09 & 6,7 & 0,59 \\
T4 & 3 & 2 & 3,68 & 0,66 & 5,3 & 0,59 \\
T5 & 4 & 3 & 4,66 & 0,84 & 5,9 & 0,56 \\
T6 & 7 & 4 & 7,29 & 2,7 & 8 & 0,97 \\
T7 & 4 & 1 & 3,61 & 0,26 & 6,8 & NaN \\
\hline
\end{tabular}

optimale V de tâche en tâche ou globalement.

$$
V=\left(\frac{E R_{S 2}-E R_{S 1}}{E R_{S 1}}\right) * 100
$$

L'intérêt est encore une fois de ramener sur une même échelle les différences entre les écarts et ainsi de les rendre comparables. Nous quantifions ainsi la progression tout en la rendant comparable entre tâches et entre logiciels. Lorsque V est positif, cela veut dire que l'écart relatif de la seconde tâche est supérieur à celui de la tâche précédente, donc que la performance du sujet se dégrade. Dans le cas d'une variation négative, la performance se rapproche de l'optimal dans la seconde tâche. Comme les écarts relatifs, cette variation peut être calculée globalement ou par type de complexité permettant ainsi de mieux caractériser la nature des progrès.

Les résultats présentés dans la figure 1 permettent d'observer que la progressions n'est pas linéaire pour aucun des deux logiciels, ce qui est compréhensible dans la mesure où (i) les sujets n'ont pas été initiés préalablement à l'utilisation du logiciel, et (ii) les tâches ne sont pas ordonnées selon les écarts relatifs, mais selon un premier indice de complexité qui s'avère finalement insatisfaisant. On observe cependant que les sujets progressent entre toutes les tâches, sauf entre les tâches $4>5$ et $5>6$ ce qui s'explique par le fait que la gradation de la complexité était mal calibrée avec notre premier indice. Lorsqu'ils progressent, les sujets utilisant HMK présentent un meilleur score de variation que ceux utilisant LO, sauf entre les tâches $2>3$. De même, lorsque la variation est positive, elle est de moindre ampleur pour les utilisateurs de HMK.

La variation globale entre les tâches $1>7$ (Figure 1. a) montre une progression avec les deux éditeurs. Cette variation est respectivement de $-0,398$ avec LO contre $-0,60$ avec HMK. Les sujets apprennent donc bien l'utilisation du logiciel dans les deux cas, mais cet apprentissage est plus efficient avec HMK. L'IC du nombre d'action selon l'éditeur ((Figure 1. b) nous indique que pour les tâches 1 à 4 , les élèves ayant utilisé HMK ont utilisé des procédures plus proches de la procédure optimale. Concernant les tâches 5 à 7 on observe des résultats inverses qui s'explique du fait que les participants ayant utilisé uniquement LO n'ont pas mené à terme les tâches. Enfin, concernant les IC nombre de switches (Figure 1c) et l'IC nombre de changement de fenêtre (Figure 1d), on peut constater que pour l'ensemble des tâches les élèves ayant eu recours au logiciel HMK ont procédé à moins de changement de fenêtre et moins de switches.

\section{DISCUSSION ET CONCLUSION}

Nos résultats de cette première étude exploratoire [3] montrent que la procédure utilisée au départ par les sujets est plus proche de l'optimal avec HMK que pour LO. Bien que les tâches soient organisées de façon croissante, la différence de complexité entre la procédure optimale et la procédure observée décroit plus vite avec HMK qu'avec LO ce qui atteste qu'il est plus facile de s'approprier le premier éditeur. Ces différences sont surtout marquées pour la complexité de planification $\left(\mathrm{IC}_{\mathrm{A}}\right)$ et la gestion des fenêtres $\left(\mathrm{IC}_{\mathrm{F}}\right)$.

Lors de cette première étude, le décompte des opérations nécessaires au calcul des indices de complexité a été réalisé manuellement en annotant l'enregistrement vidéo des passations. Or, les indices de complexité que nous venons de présenter reposent entièrement sur des opérations produisant un résultat identifiable dans le dispositif informatique. Ces éléments présentent des avantages notamment pour l'informatisation du décompte de ces opérations afin de minimiser le risque d'erreur et faciliter l'analyse mais aussi pour une mesure des temps, pour chaque action, et globaux beaucoup plus précise.

L'automatisation du recueil exhaustif et précis des actions réalisées par l'utilisateur doit également permettre de construire d'autres indices, mais surtout, à l'image de ce qui a été fait en résolution de problème, de caractériser l'organisation de l'activité et les révisions des représentations identifiables par les pauses dans l'activité et le changement d'organisation de l'activité [15]. On peut ainsi étudier de manière fine l'instrumentalisation du logiciel par les sujets. De plus, cet outil métrique permet d'étudier l'apprennabilité d'un logiciel quel qu'il soit, dans le domaine de la saisie (clavier virtuel pour la saisie de texte, écriture phonétique ou encore par pictogrammes).

Les résultats présentés dans cette étude permettent de mettre en évidence que si l'indice de complexité utilisé dans la précédente étude [3] est efficient pour comparer les sujets entre eux, celui-ci ne permet pas de comparer les tâches entre elles. C'est pourquoi nous préférons calculer l'écart relatif à la procédure optimale $\mathrm{ER}_{\mathrm{T}}$ qui semble être un meilleur critère pour ordonner les tâches. La même démarche permet également de résumer la procédure utilisée par les sujets en calculant ERs. Cet indice permet les comparaisons intrasujets et intertâches, même si ces dernières sont de complexités différentes. Il est alors plus intéressant de faire les comparaisons sur $\mathrm{ER}_{\mathrm{S}}$ plutôt que sur $\mathrm{IC}_{\mathrm{G}}$, notamment pour mesurer la variation entre tâche de l'efficience. Cela nous a permis d'observer que la variation 

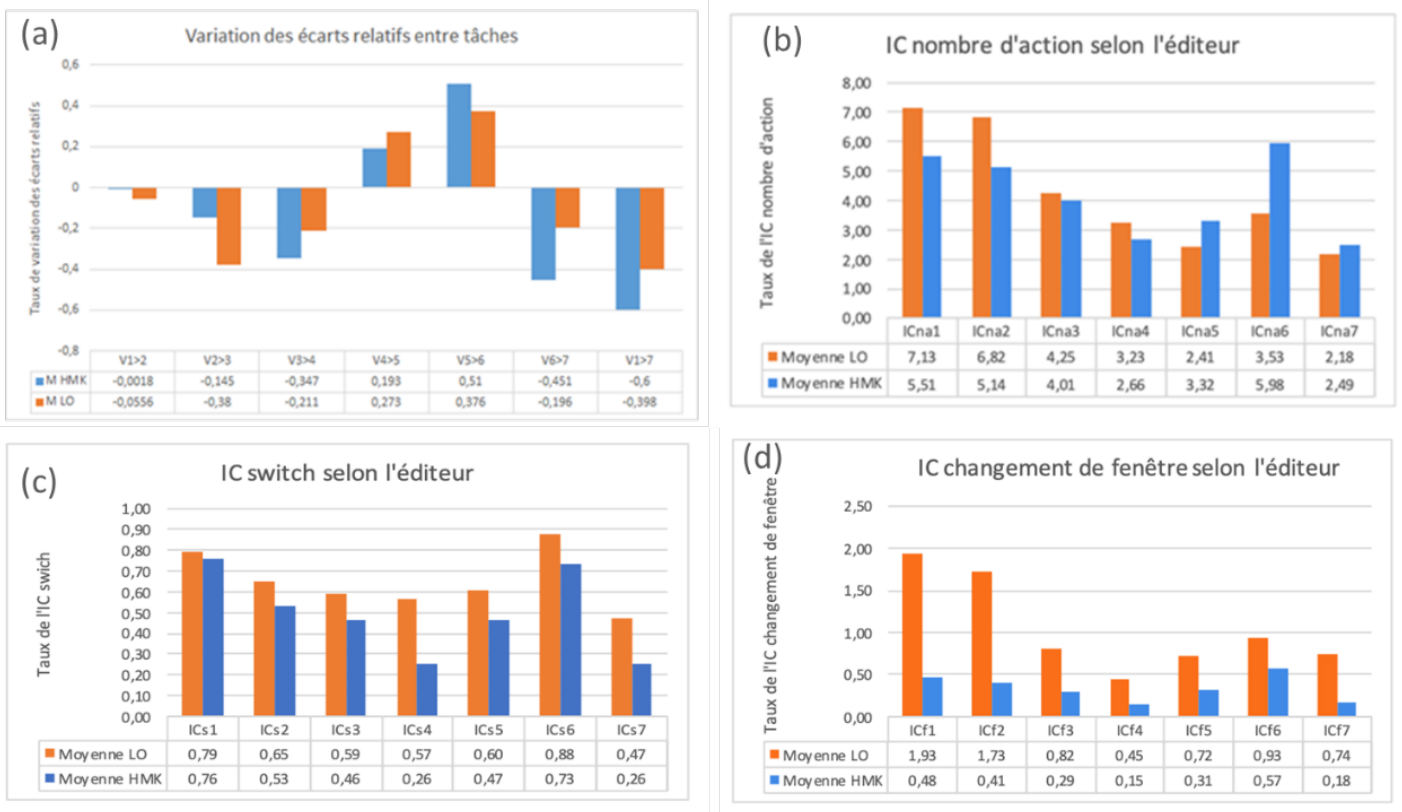

Figure 1: : Résultats de l'étude pour les différents indicateurs

n'est pas linéaire et de nous interroger sur l'ordre de présentation des tâches comme nous l'avons fait.

$\mathrm{ER}_{\mathrm{S}}$ facilite également l'identification des sujets qui n'ont pas mené la tâche à leur terme dès l'instant où leur nombre d'actions est inférieur à la procédure optimale. Ce critère à lui seul n'est cependant pas suffisant et doit être complété par d'autres critères. Ces précautions permettent de s'assurer que la tâche effective des sujets correspond bien à la tâche prescrite, condition indispensable à la comparabilité de l'activité entre les deux logiciels.

Par ailleurs, les écarts relatifs et les taux de variation entre tâches offrent la possibilité d'étudier par des régressions linéaires l'évolution des performances. Cela nous permettrait, éventuellement, de voir quelles tâches seraient prédictives de la performance aux tâches suivantes. Et ainsi, nous pourrions avoir une analyse plus fine des progressions.

Enfin, notre approche ne nécessite pas de connaître de manière exhaustive l'espace de la tâche, mais simplement de définir les bornes pour pouvoir calculer les indices que nous venons de présenter. Elle est surtout suffisamment générale pour être appliquée à l'analyse d'autres logiciels. Notre approche ne permet pas d'appréhender la part de l'activité qui ne produit pas d'effet dans l'interface, comme l'orientation du regard ou ce que le sujet pourrait faire sur son bureau en utilisant par exemple des notes manuscrites. Le recueil des données informatiques devra donc être complétée par d'autres dispositifs d'observation.

\section{REMERCIEMENTS}

Les auteurs remercient les élèves qui ont participé à cette expérimentation.

\section{RÉFÉRENCES}

[1] Brossard-Racine, M., Majnemer, A., Shevell, M., Snider, L., \& Belanger, S. A. 2011. Handwriting capacity in children newly diagnosed with attention deficit hyperactivity disorder. Research in Developmental Disabilities, 32(6), 2927-2934. http://dx.doi.org/ 10.1016/j.ridd.2011.05.010.

[2] Karatekin, C., Markiewicz, S. W., \& Siegel, M. A. 2003. A preliminary study of motor problems in children with attention-deficit/hyperactivity disorder. Perceptual and Motor Skills, 97(3), 1267-1280. https://doi.org/10.2466/pms.2003.97.3f.1267

[3] Baudet, R., 2020. Test d'un artefact numérique pour la saisie de formule mathématique auprès d'élèves du secondaire porteurs d'un trouble moteur. Mémoire de maitrise, Université Paris 8.

[4] Sauzin, D. 2017. Apport d'une conception centrée utilisateur adaptée selon les besoins des personnes en situation de handicap. Thèse (2017), Discipline ou spécialité: Informatique, Université Toulouse 3, Paul Sabatier.

[5] Vigouroux, N., Sauzin, D., Vella, F., \& Dubus N. 2017. Les médiateurs medicoeducatifs au centre de la conception : l'interface HMK pour les collégiens et lycéens (short paper). Dans : Conférence francophone sur l'Interaction HommeMachine (IHM 2017), Poitiers, 29/08/2017-01/09/2017, ACM, p. 8, (support électronique). https://doi.org/10.1145/3132129.3132151

[6] Smits-Engelsman, B.C.M., van Galen, G.P., 1997. Dysgraphia in children: lasting psychomotor deficiency or transient developmental display. Journal of Experimental Child Psychology 67, 164-184. https://doi.org/10.1006/jecp.1997.2400

[7] Paz-Villagran, V., Gilhodes, J. \& Velay, J. 2012. Les enfants dysgraphiques sont-ils réellement plus lents que les autres? Dev́eloppements, 13(4), 38-44. doi:10.3917/ devel.013.0038.

[8] Vella, F. Dubus, N., Sauzin, D., Bertrand, E., Gallard, C., Malet, C., Groleau, E., Vigouroux, N. \& Preel, W. 2018. La conception d'un clavier numérique conçu par des enseignants en mathématiques et des ergothérapeutes, peut-elle favoriser son adoption par des élèves en situation de handicap : le cas de HandiMathKey. (2018) In: Ludovia (2018), 20 August 2018 - 23 August 2018 (Ax-Les-Thermes, France).

[9] MacKenzie, I.S. 2002. KSPC (keystrokes per character) as a characteristic of text entry techniques, Proceedings of the Fourth International Symposium on HumanComputer Interaction with Mobile Devices. Heidelberg, Germany: SpringerVerlag

[10] Martin, B., \& Pecci, I. 2007. État de l'art des claviers physiques et logiciels pour la saisie de texte. Revue d'interaction homme-machine, vol. 8, $\mathrm{n}_{0}^{50} 2$ 2, 147-205. http: //europiaproductions.free.fr/RIHM/V8N2/8-RIHM-Martin-Pecci\%20PDF.pdf

[11] Agnès, A., Fleury, S., Auzerais, A., Bisson, I., Dulau, E., Buisine, S., \& Richir, S. 2019. Complementarite des outils de saisie de texte en environnement virtuel immersif. 11. http://hdl.handle.net/10985/15975

[12] Richard, J.-F. 1999. Comportements, buts et représentations. Psychologie Française, 44, 75-90.

[13] Megalakaki, O., Tijus, C., Baiche, R., \& Poitrenaud, S. 2012. The effect of semantics on problem solving is to reduce relational complexity. Thinking \& Reasoning, 
18(2), 159-182. https://doi.org/10.1080/13546783.2012.663101

[14] Clément, E. 2005. Compréhension et résolution de problème: Que nous apprennent les difficultés de l'apprenant. Rééducation orthophonique, 25, 239-250. https://www.academia.edu/4628958/COMPREHENSION_ET_RESOLUTION_ DE_PROBLEME

[15] Clément, E. 2009. La résolution de problème: A la découverte de la flexibilité cognitive. Armand Colin.
[16] Lee, H., Gambette, Ph. \& Barkat-Defradas, M. 2014. iPhocomp : calcul automatique de l'indice de complexitéṕhonétique de Jakielski. Actes de la XXXe' Édition des fournées d' Études sur la Parole, Juin 2014, Le Mans, 662-630. https://core.ac.uk/ download/pdf/48317965.pdf

[17] Gabadinho, A., Ritschard, G., Müller, N-S., Studer, M. 2011. Analyzing and Visualizing Sate Sequences in R with TraMineR. Journal of Statistical Software 40(4):1-37. https://doi.org/10.18637/jss.v040.i04 